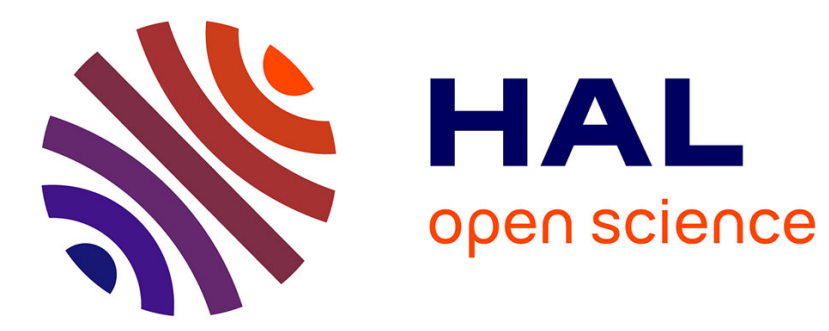

\title{
De la politisation à l'instrumentation d'un outil de gestion: le cas du budget dans les théâtres associatifs
}

Fabienne Villesèque-Dubus, Pascale Amans, Agnès Mazars-Chapelon

\section{To cite this version:}

Fabienne Villesèque-Dubus, Pascale Amans, Agnès Mazars-Chapelon. De la politisation à l'instrumentation d'un outil de gestion: le cas du budget dans les théâtres associatifs. Revue management \& avenir, 2010, 40, pp.60-79. halshs-00676921

\section{HAL Id: halshs-00676921 https://shs.hal.science/halshs-00676921}

Submitted on 6 Mar 2012

HAL is a multi-disciplinary open access archive for the deposit and dissemination of scientific research documents, whether they are published or not. The documents may come from teaching and research institutions in France or abroad, or from public or private research centers.
L'archive ouverte pluridisciplinaire HAL, est destinée au dépôt et à la diffusion de documents scientifiques de niveau recherche, publiés ou non, émanant des établissements d'enseignement et de recherche français ou étrangers, des laboratoires publics ou privés. 


\title{
De la politisation à l'instrumentation d'un outil de gestion : le cas du budget dans les théâtres associatifs ${ }^{1}$
}

\author{
Pascale Amans \\ LGC \\ Université Toulouse 3 \\ IUT «A $»$ Paul Sabatier \\ Département GEA \\ 115 route de Narbonne \\ 31077 Toulouse Cedex 4 \\ pascaleamans@ hotmail.com
}

\author{
Agnès Mazars- \\ Chapelon \\ CREGOR - MRM \\ Université Montpellier 2 \\ IUT de Montpellier \\ Département informatique \\ 99 avenue d'Occitanie \\ 34296 Montpellier Cedex 5 \\ agnes.mazars-chapelon@univ- \\ montp2.fr
}

\author{
Fabienne Villesèque- \\ Dubus \\ CREGOR - MRM \\ Université Montpellier 2
}

IAE de Perpignan

Université de Perpignan

52 avenue Paul Alduy

66860 Perpignan Cedex

fabienne.villeseque@ univ-perp.fr

Pascale Amans, diplômée de l'École Normale Supérieure de Cachan, agrégée du second degré en économie et gestion, est maître de conférences en sciences de gestion. Ses recherches, menées en contrôle, portent notamment sur l'identité et la performance des organisations, ainsi que sur la coopération. Plusieurs de ses travaux sont conduits dans le secteur artistique et culturel.

Agnès Mazars-Chapelon, diplômée de l'ESSEC, est maître de conférences en sciences de gestion. Elle s'intéresse dans le cadre de ses activités de recherche aux questions de contrôle et de dynamique des organisations, plus particulièrement à travers leurs outils de gestion, leurs inter-relations et leur origine, notamment dans leur dimension cognitive et émotionnelle.

Fabienne Villesèque-Dubus, agrégée du second degré en économie et gestion, est maître de conférences HDR en sciences de gestion à l'IAE de Perpignan. Ses recherches, menées en contrôle de gestion, portent sur les différents rôles des systèmes et outils de contrôle, et notamment sur ceux associés au système budgétaire. Elle s'intéresse en particulier aux interactions intra et inter-organisationnelles liées à la mise en œuvre des outils de contrôle.

\section{Résumé}

Parmi les utilisations du budget par les associations du spectacle vivant, quelles sont celles qui relèvent d'un usage politique externe dans le cadre des relations avec les collectivités, celles qui correspondent à un usage instrumental interne et quel est le «dosage » entre ces deux types d'utilisations? Telle est la question à laquelle cet article répond, d'abord de manière théorique, puis de manière pratique, au moyen de l'étude de deux cas de théâtres associatifs.

\begin{abstract}
This article deals with the ways performing arts societies use budgets. It aims at answering the following questions: amongst the ways in which these societies use their budget, which ones relate to political external uses in the context of their relations with local government, which ones concern internal implementation uses, and what is the ratio between those two types of uses? The answer is based on the study of two local drama societies.
\end{abstract}

\footnotetext{
1 AMANS P., MAZARS-CHAPELON A. et VILLESEQUE-DUBUS F. (2010), « De la politisation à l'instrumentation d'un outil de gestion : le cas du budget dans les théâtres associatifs », Management \& Avenir, n²0, décembre, pp. 234-253.
} 


\section{De la politisation à l'instrumentation d'un outil de gestion : le cas du budget dans les théâtres associatifs}

Les collectivités telles les mairies ont dans leurs missions de service public un volet artistique et culturel. Ne pouvant accomplir seules cette mission, elles en confient en partie la réalisation à d'autres organisations ou soutiennent des établissements dont le projet est en accord avec cette mission. Parmi ces organisations figurent les associations, qui peuvent être vues comme des partenaires précieux des mairies dans la mise en ouvre des politiques publiques locales (Fabre, 2005a, 2006). Les associations de leur côté ont besoin du soutien financier des collectivités pour mener à bien leurs activités et ainsi accomplir leur mission; elles doivent se rapprocher de ces dernières pour assurer leur existence et leur devenir (Ragi, 2000). La contribution des associations artistiques et culturelles à l'accomplissement d'une mission de service public et les financements publics qui en découlent justifient le contrôle que ces collectivités exercent sur ces associations; et ce, d'autant plus dans un contexte de réforme des collectivités publiques locales qui vise notamment à permettre une meilleure allocation des moyens financiers de ces dernières ${ }^{2}$. Plusieurs auteurs ont d'ailleurs travaillé sur la question du contrôle des associations -non exclusivement culturelles- par les collectivités et sur celle, liée, des outils susceptibles de servir aux collectivités pour effectuer ce contrôle (Lande, 2001 ; Fabre, 2004, 2005a, 2005b, 2006 ; Zoukoua, 2006, 2008 ; Chekkar et Zoukoua, 2009...). Ce qui nous intéresse ici, c'est de nous placer principalement non plus du point de vue des collectivités, mais de celui des associations et de voir comment ces dernières utilisent l'un des instruments de ce contrôle, le budget.

L'examen de la littérature sur les budgets souligne les multiples modes d'utilisation du budget, au sein desquels l'on observe notamment une double dimension, politique et instrumentale, de ce système de contrôle (Burchell et al., 1980 ; Covaleski et Dirsmith, 1983, 1986, 1988a, 1988b ; Berland, 1999 ; Sponem, 2004 ; Amans et Villesèque-Dubus, 2008...). Dans le cas des associations du spectacle vivant, du fait des spécificités de ces dernières, le budget serait un outil avant tout politique, contrairement à son mode de fonctionnement dans les entreprises. Le terme «politique » est ici à entendre dans une acception proche de son sens étymologique -polis signifie cité, ville- et aristotélicien. Aristote voit en effet l'homme comme un animal politique, c'est-à-dire comme un être fait pour construire une vie dans une société, société au sein de laquelle il est lié aux autres hommes par le langage, le discours. Les utilisations du budget qui sont ici qualifiées de politiques sont par conséquent celles nées de l'ancrage de l'association dans une société composée notamment de collectivités qui financent cette association et de la nécessité pour cette dernière de communiquer avec ces collectivités. Elles sont donc constituées par l'ensemble des utilisations du budget à visée externe. A contrario, les utilisations instrumentales sont dans cet article des utilisations internes aux associations. Né de la relation entre association et financeurs, et en particulier de la relation entre association et collectivités -une grande partie du financement des associations provenant de partenaires publics-, le budget serait principalement conçu et utilisé par les associations à des fins externes, en direction des collectivités et en fonction des attentes perçues de ces dernières. L'origine externe de ce budget politique est un point qu'il a en commun avec le budget administratif évoqué par Chatelain (1998), lequel se traduit essentiellement par un mécanisme d'allocation de fonds.

2 Source : Site Internet du Ministère de l'Intérieur, de l'Outre-mer et des collectivités territoriales. http://www.interieur.gouv.fr/sections/reforme-collectivites, consulté le 31 août 2010. 
Cet article a pour objectif de mieux cerner les utilisations possibles du budget par les associations du spectacle vivant sous l'angle d'une double dimension, politique externe et instrumentale interne et, en particulier, de prendre la mesure de la part relative de ces deux dimensions. Parmi les utilisations du budget par ces associations, quelles sont celles qui relèvent d'un usage politique externe dans le cadre des relations avec les collectivités, celles qui correspondent à un usage instrumental interne et quel est le «dosage » entre ces deux types d'utilisations ? Telle est la question de recherche à laquelle cet article vise à répondre.

Cette question étant peu explorée dans la littérature en contrôle, notre démarche relève de l'abduction (Glaser et Strauss, 1967), avec des allers-retours successifs entre d'une part la littérature portant sur les budgets ainsi que sur la gestion des associations du spectacle vivant, d'autre part le terrain. Deux cas de théâtres associatifs financés par des mairies ont ainsi été étudiés, en s'appuyant à titre principal sur des entretiens semi-directifs centrés.

La première partie de l'article présente le cadre théorique de cette recherche ; la seconde partie est consacrée à la présentation de la méthode de recherche ainsi qu'à la restitution et à la discussion des résultats.

\section{1- Du contrôle au budget dans les associations du spectacle vivant : quelle lecture théorique?}

Cette première partie pose le cadre théorique de cette recherche, celui du contrôle des associations du spectacle vivant. Nous partons des éléments de contradiction ou de cohérence théoriques entre contrôle et associations du spectacle vivant, pour ensuite nous appuyer sur le cas du budget comme mode de contrôle particulier de ce type d'organisation ; nous mettons alors en exergue les utilisations théoriques du budget dans les associations du spectacle vivant.

\section{1- Contrôle et associations du spectacle vivant : quels paradoxes théoriques ?}

Les spécificités des associations du spectacle vivant peuvent sembler a priori contradictoires avec la gestion et le contrôle. Cette section, qui ne prétend pas à l'exhaustivité, souligne quelques paradoxes théoriques entre d'une part les caractéristiques de ces associations, d'autre part la gestion et le contrôle. Ces paradoxes découlent de l'appartenance au secteur des associations artistiques et culturelles en général; ils sont exacerbés dans le cas des associations du spectacle vivant du fait de la dimension fortement créatrice de ces dernières.

Quelques premiers paradoxes découlent de l'appartenance au secteur des associations artistiques et culturelles. L'appartenance à l'ensemble des «entreprises artistiques et culturelles » (Evrard et Chiapello, 2004, p. 8) entraîne certaines spécificités au niveau de «la définition des objectifs », de «la nature [des] produits» ainsi que du «poids de l'intervention de la puissance publique ». Dès lors, leur gestion et en particulier leur contrôle sont empreints de paradoxes, qui se résument par autant de questions.

Tout d'abord, l'existence d'une finalité artistique et culturelle fait que, quel que soit le statut juridique d'une organisation de ce secteur, sa mission ne se réduit pas à la réalisation d'un profit : d'où une dualité axiologique (Abdallah, 2007) entre les préoccupations artistiques et celles relatives à la rentabilité et, plus généralement, entre les préoccupations artistiques et celles que l'on pourra qualifier d'économiques, de gestionnaires ou encore de financières (celles par exemple relatives à la maîtrise des coûts). L'antinomie entre ces deux dimensions, l'artistique d'une part, l'économie et la gestion d'autre part, est d'ailleurs «utilisée par les professionnels et le public pour plaider le maintien de conduites affranchies de considérations économiques» (Bonnafous-Boucher, Chatelain-Ponroy, Evrard et Mazallon, 2003, p. 169). L'on peut s'attendre à ce que cette prévalence des considérations artistiques 
soit particulièrement prononcée dans les associations du fait de leur caractère non lucratif. Dans ces organisations comme dans l'ensemble des organisations publiques et non marchandes, la définition de la performance se fonde sur la déclinaison en objectifs d'une mission parfois floue et ambiguë et donc difficilement opérationalisable (Amans et RascolBoutard, 2008). Dès lors, leur contrôle se heurte à un double paradoxe, lequel se traduit par l'interrogation suivante : comment contrôler des organisations dont la finalité est floue et les objectifs mal identifiés ?

En outre, la nature des produits du secteur artistique et culturel est particulière. Ainsi, si la diffusion de certains produits culturels peut s'appuyer sur des processus de réplication, l'activité de production quant à elle correspond à une production de prototypes (Assassi, 2003). D'où le paradoxe et l'interrogation associée : comment contrôler des exceptions ?

Enfin, les collectivités locales sur le territoire desquelles se trouvent les associations artistiques et culturelles sont parties prenantes de ces dernières, qu'en pratique elles participent souvent largement à financer. Ces financements publics apparaissent comme une condition de la diffusion et de la production artistique (Agid et Tarondeau, 2003, p. 105). Comment, quand glissent ainsi objectifs et moyens d'une entité, la collectivité, à une autre, l'association artistique et culturelle, déterminer les frontières de ces organisations ? Se pose par conséquent cette autre question, paradoxale : comment contrôler des organisations dont on ne cerne pas les frontières, non délimitées? Par exemple, calculer des coûts dans de telles organisations est problématique (Burlaud et Simon, 2000 ; Chatelain-Ponroy, 2001, 2003).

Ces paradoxes théoriques deviennent encore plus saillants dans le cas des associations oeuvrant dans le domaine du spectacle vivant, à forte composante créatrice. Le spectacle vivant peut en effet être défini comme "la rencontre physique entre des interprètes, un public et une cuvre artistique" (Robin, 1992). [Il] fait donc référence aux arts de la scène » (Assassi, 2003, p. 130). L'activité des organisations étudiées, qui consiste en une création artistique, paraît a priori incompatible avec les préoccupations économiques et gestionnaires en général et avec le contrôle en particulier. Les organisations auxquelles nous nous intéressons ici sont plus confrontées que d'autres du même secteur à la potentielle contradiction entre contrôle et art. En effet, le contrôle, défini comme des "influences créatrices d'ordre, d'une certaine régularité » (Chiapello, 1999, p. 198) et donc impliquant l'idée de maîtrise, peut paraître incompatible avec la création artistique. Tous deux sont " $a$ priori pareils à l'eau et au feu » (Chiapello, 1994, p. 9). Plus précisément, mettre en scène des spectacles vivants nécessite de travailler avec des artistes vivants, a priori peu favorables au contrôle et plus généralement à la gestion. Deux points en effet sont très sensibles chez les artistes : leur revendication d'autonomie et leur rapport à l'argent (Chiapello, 1998). Ainsi, les procédures et le contrôle budgétaire sont vus comme des contraintes qui restreignent l'autonomie des artistes (Benghozi, 1990). Vouloir contrôler une association du spectacle vivant, c'est vouloir faire cohabiter des éléments a priori incompatibles : le contrôle d'une part, la création artistique et les artistes vivants d'autre part.

En outre, le spectacle vivant correspond plus que d'autres activités artistiques et culturelles à une activité de prototypes (Assassi, 2003).

Pourtant, le travail artistique n'échappe pas à tout mode de contrôle (Chiapello, 1999) et la gestion apparaît même comme une condition de la création (Le Theule, 2008). La question posée dans ces organisations est donc moins celle de l'opposition entre logique artistique et logique économico-financière que celle de leur nécessaire réconciliation (Bathurst, Williams et Rodda, 2007). D'où le paradoxe : les organisations du spectacle vivant, organisations artistiques a priori théoriquement incontrôlables, sont paradoxalement contrôlées. 
Sur la base des paradoxes et spécificités mis en lumière ci-dessus, l'on peut s'attendre à ce qu'un contrôle de ces organisations fondé sur des prévisions et sur un suivi comprenant une comparaison de ces prévisions aux réalisations soit problématique. C'est ce que montre la section suivante, qui propose une approche théorique d'un outil emblématique du contrôle formel, le budget. L'on peut également s'attendre, compte tenu de ce que les associations du spectacle vivant évoluent dans un environnement fortement institutionnalisé, entourées de collectivités parties prenantes souvent largement contributrices sur le plan financier, avec lesquelles elles entretiennent des relations étroites, à ce que ces associations utilisent le budget principalement en direction des collectivités à des fins de communication et de légitimation par rapport aux attentes supposées de ces dernières. Ces utilisations du budget, que nous qualifierons de politiques, sont également développées ci-dessous.

\section{2- Une lecture théorique des utilisations du budget dans les associations du spectacle vivant}

La littérature en contrôle souligne que le budget, outil de gestion, peut jouer d'autres rôles qu'instrumentaux, sociaux et politiques notamment (Berland, 1999). Ainsi, nombre de définitions du budget associent, à côté des rôles instrumentaux de l'outil, des fonctions que les auteurs présentent comme davantage stratégiques, coordinatrices, de communication (Bouquin, 1998), d'animation et psychosociologiques (Gervais, 1997), humaines (GignonMarconnet, 2003), interactives (Simons, 1990, 1995), légitimatrices et politiques (Burchell et al., 1980 ; Covaleski et Dirsmith, 1983, 1986, 1988a ; 1988b).

Cette section n'a pas vocation à proposer une synthèse de la littérature balayant l'ensemble des fonctions possibles du budget, mais à apporter un éclairage théorique sur deux grandes familles de fonctions du budget, particulièrement intéressantes dans le type d'organisations étudiées compte tenu des spécificités de ces dernières. Seront ainsi présentées d'abord les fonctions politiques externes $\mathrm{du}$ budget dont on s'attend compte tenu du degré d'institutionnalisation de l'environnement à ce qu'elles jouent pleinement. Ces organisations évoluent en effet dans un environnement marqué par une forte dépendance vis-à-vis des organismes financeurs, ce qui peut en particulier, nous le verrons, les conduire à adopter certaines pratiques de gestion en conformité avec les attentes de leur environnement. Seront ensuite évoquées les fonctions instrumentales internes dont on s'attend comme on l'a vu plus haut à ce qu'elles soient plus problématiques.

Suivant Burchell et al. (1980), les budgets pourraient occuper de nombreuses fonctions, audelà de leurs fonctions instrumentales. Les auteurs évoquent en particulier des fonctions davantage politiques, de «machine politique » et de « machine à rationaliser » notamment, dans une perspective de légitimation des actions (Burchell et al., 1980). Cette dernière fonction rationaliste et légitimatrice est en particulier fondée à s'exercer dans une perspective externe, dans les relations entretenues avec les partenaires financiers notamment, pouvant aller même jusqu'à prendre une dimension symbolique ou rituelle (Meyer et Rowan, 1977).

Les théories néo-institutionnelles permettent d'expliquer ce mode d'utilisation dans une perspective externe au-delà de la recherche d'efficience interne, les organisations utilisant alors le budget pour se conformer à un ensemble de règles et pressions sociales caractéristiques de leur environnement (Meyer et Rowan, 1977 ; Burchell et al., 1980). Les auteurs soulignent en particulier l'importance du budget pour permettre de comprendre l'émergence des actions et pour justifier les choix entrepris. L'outil permettrait ainsi de rationaliser les choix a posteriori, ce qui est d'autant plus important que les intérêts externes sont fortement présents dans l'organisation. Dans cette perspective, les budgets et plans 
pourraient alors être construits et orientés en fonction des attentes externes perçues et en vue d'afficher une certaine rationalité.

Comme Meyer et Rowan (1978), repris par Burchell et al. (1980, p.18), le soulignent : "la vie dans les organisations modernes est un inter-jeu constant entre les activités que nous devons mener à bien et les comptes organisationnels que nous devons rendre ».

Suivant cette perspective néo-institutionnelle, les budgets pourraient constituer des «mythes rationnels », donnant l'illusion de la rationalité (Meyer et Rowan, 1977 ; Sponem, 2004). D'où des processus d'isomorphismes, des organisations adoptant certaines pratiques en vue de montrer qu'elles sont de «bonnes organisations » et surtout qu'elles agissent en conformité avec leur environnement et dans le but de satisfaire des exigences extérieures (DiMaggio et Powell, 1983 ; Abrahamson, 1996 ; Gehrke et Zarlowski, 2003 ; Rojot, 2005) réelles ou supposées.

En complément des travaux propres aux aspects légitimateurs et post-rationalisateurs des budgets, ces derniers sont aussi vus comme des moyens de négociation, plus précisément avec les partenaires externes. Meyer et Rowan (1977), ainsi que Covaleski et Dirsmith (1983, 1986, 1988a, 1998b), ont souligné l'intérêt d'utiliser des budgets afin de négocier avec les parties prenantes dans un environnement fortement institutionnalisé.

Une telle grille de lecture semble particulièrement intéressante pour étudier le cas des associations du spectacle vivant, lesquelles développent des relations privilégiées avec leurs partenaires externes, bailleurs de fonds tout particulièrement, parmi lesquels les collectivités. Dès lors, et compte tenu également des spécificités de ces associations, une utilisation des budgets en interne pour le contrôle est-elle envisageable ?

Selon Bruns et Waterhouse (1975) repris par Covaleski et Dirsmith (1983), «les budgets sont définis comme des plans financiers fournissant une base pour diriger et évaluer la performance des individus et sous-unités dans l'organisation de manière à mieux coordonner et contrôler leurs différentes activités ». D'un point de vue instrumental, le budget remplit ainsi deux fonctions principales, liées: une fonction d'allocation et de prévision des ressources qui s'inscrit dans le cadre de la mission de planification des budgets, ainsi qu'une fonction plus ancrée sur le contrôle des résultats et les calculs d'écarts. Cette dernière mission, dite de surveillance et de contrôle, s'inscrit dans une approche «classique » du contrôle tel que le définissait Anthony (1965), ancrée sur la vérification de l'atteinte des objectifs de l'organisation, laquelle passe par la recherche d'efficacité et d'efficience.

L'allocation des ressources, qui forme l'une des premières étapes du processus budgétaire, concernerait donc a priori tout type d'organisation, y compris culturelle. Cependant, l'incertitude caractérisant la définition des objectifs des associations du spectacle vivant ainsi que les résultats de la création artistique -processus dont l'issue est empreinte d'incertitude (Assassi, 2008)- conduit à une situation paradoxale. Celle-ci peut se résumer par l'interrogation suivante : comment prévoir des ressources quand les objectifs de l'organisation sont flous et le résultat de la production incertain ? Par ailleurs, si l'idée de création repose sur l'hypothèse que la norme n'est pas préétablie (Chiapello, 1997), comment établir des prévisions de normes?

C'est en particulier sur ces prévisions que par la suite va s'appuyer le système de suivi et de contrôle des résultats. Ce contrôle s'inscrit dans le cadre de la fonction diagnostique des budgets, au sens de Simons, lequel définit les contrôles de diagnostic comme les "systèmes d'information formels que les managers utilisent pour surveiller les résultats organisationnels et corriger les écarts par rapport aux standards prévus de performance » (Simons, 1995, p. 59). Tout système d'information formel, y compris le budget, serait ainsi utilisable à des fins de diagnostic s'il est possible de définir des objectifs par avance, mesurer les résultats, 
calculer des écarts de performance et utiliser les informations sur les écarts pour instaurer un contrôle par rétroaction. Cependant, l'incertitude pesant sur la création artistique peut constituer un obstacle à la mise en œuvre de ce principe : comment mettre en évidence des écarts ayant un sens et les interpréter alors que les prévisions de résultats sont difficiles à établir?

Cette première partie de l'article a permis de présenter le cadre de réflexion support de notre recherche. Des paradoxes théoriques relatifs au contrôle des associations du spectacle vivant et à deux grands types de rôles possibles du budget dans ces organisations ont été mis en évidence. D'un point de vue théorique, si une première lecture peut mettre l'accent sur une contradiction fondamentale entre contrôle budgétaire et associations du spectacle vivant, il apparaît surtout que le cœur du paradoxe se situe principalement dans la dimension instrumentale interne plus que dans la dimension politique externe du budget. En effet, si d'un point de vue théorique les spécificités des associations semblent favoriser le développement d'utilisations politiques externes $\mathrm{du}$ budget, elles paraissent également difficilement compatibles avec des utilisations plus instrumentales et internes de ce dernier. Qu'en est-il en pratique ? Comment les utilisations politiques externes inscrites dans le cadre des relations avec les collectivités ainsi que les utilisations plus instrumentales en interne sont-elles mises en œuvre par les associations du spectacle vivant et quelle est la part relative de ces utilisations ? La littérature a permis de fournir des pistes de réflexion que nous avons souhaité analyser et approfondir par une étude de terrain. Cette dernière est évoquée dans la partie suivante, laquelle propose une lecture pratique des rôles du budget dans deux associations artistiques et culturelles.

\section{2- Des rôles politiques aux rôles instrumentaux du budget dans les associations du spectacle vivant : quelle lecture pratique ?}

Cette partie s'attache à restituer la démarche de recherche adoptée pour mener à bien l'étude de terrain, ainsi que les enseignements qui en découlent.

\section{1- Méthode de recherche}

Dans cette section sont détaillées les principales étapes de la recherche et présentés les cas étudiés.

Nous avons centré notre étude sur le budget comme lien entre théâtres associatifs et mairies. En effet, la diversité des associations du spectacle vivant financées par de multiples parties prenantes a conduit à resserrer l'échantillon théorique (Yin, 1990) examiné ici en nous centrant sur notre objet d'étude -le budget- et en privilégiant des associations de spectacle vivant nouant des liens étroits avec une collectivité particulière : des théâtres fortement subventionnés par des mairies qui elles-mêmes consacrent une part conséquente de leur budget culture à ces établissements. Ce cas de figure nous est apparu propice à l'étude des fonctions du budget, dans une perspective d'analyse de ses fonctions instrumentales internes et politiques externes. En particulier, dans ces établissements, le budget est, comme le résume le président de l'un des conseils d'administration, "à la base de tout»; sur le plan des relations entre mairie et association, son rôle est important puisqu'il constitue le soutien des financements pour le théâtre et qu'il permet par ailleurs au financeur de s'assurer de la bonne utilisation des fonds.

Ce choix a été conforté par une étude exploratoire menée en début de recherche sur les fonctions du budget dans les organisations artistiques et culturelles. Cette étude, fondée sur 
des entretiens auprès de directeurs et d'administrateurs de scènes de spectacle vivant ainsi que de responsables du contrôle de gestion au sein de telles organisations, dans les deux régions où se situent les théâtres associatifs dont il est question ici, a permis de mieux connaitre l'environnement culturel de ces régions et donc le cadre dans lequel évoluent les théâtres associatifs concernés. Elle a permis également la sélection de ces derniers.

A l'issue de cette phase exploratoire, le processus de recherche s'est organisé autour de deux vagues complémentaires et successives de collecte de données par entretiens, d'abord auprès des théâtres, puis auprès des conseils d'administration et des mairies, accompagnées de l'examen de données secondaires (budgets, conventions...). L'encadré 1 ci-dessous synthétise les thèmes des guides utilisés pour les entretiens.

Encadré 1 : Guides utilisés pour les entretiens en fonction du répondant

\begin{tabular}{|c|c|}
\hline Répondant & Principaux thèmes du guide d'entretien \\
\hline $\begin{array}{l}\text { Directeur du théâtre } \\
\text { Administrateur du théâtre }\end{array}$ & $\begin{array}{l}\text { Système de pilotage de l'organisation en général } \\
\text { Outils de pilotage communément utilisés } \\
\text { Budget et procédure budgétaire }\end{array}$ \\
\hline Président du conseil d'administration & $\begin{array}{l}\text { Vie du théâtre telle qu'elle est vue par le président } \\
\text { Rôle du conseil d'administration dans le } \\
\text { fonctionnement du théâtre en général } \\
\text { Budget et procédure budgétaire }\end{array}$ \\
\hline $\begin{array}{l}\text { Directeur des affaires culturelles de la } \\
\text { mairie } \\
\text { Elu en charge de la culture à la mairie }\end{array}$ & $\begin{array}{l}\text { Attentes de la mairie vis-à-vis du théâtre } \\
\text { Vie du théâtre telle qu'elle est vue par le répondant } \\
\text { Rôle de la mairie dans la vie du théâtre en général } \\
\text { Budget et procédure budgétaire }\end{array}$ \\
\hline
\end{tabular}

Dans un premier temps ont été rencontrés le directeur et l'administrateur de chacun des théâtres associatifs, afin d'éclairer la question des usages du budget par ces théâtres, à travers le double angle de lecture, politique externe d'une part, instrumental interne d'autre part. Ce sont ces acteurs en effet, qui dans les théâtres étudiés, sont les plus impliqués dans le contrôle de ces derniers, le comptable se centrant plus sur les écritures et la paye. Les entretiens ont d'abord été conduits auprès de responsables d'un premier théâtre et ont fait émerger des résultats surprenants au regard de la littérature : certes la dimension politique externe du budget était présente mais les utilisations instrumentales en interne étaient paradoxalement elles aussi bien développées. Voulant savoir s'il s'agissait d'une exception, nous avons alors mené des entretiens dans un autre théâtre où non seulement la dimension instrumentale interne s'est avérée également très présente, mais où les utilisations politiques externes du budget sont apparues plus en retrait que dans le premier établissement. Finalement, dans deux établissements assez proches sur le plan de la taille et de la nature de la programmation, la configuration dimension politique externe/dimension instrumentale interne était assez différente pour qu'une mise en parallèle soit intéressante. Les thèmes abordés au cours de cette première vague d'entretiens réalisés auprès des théâtres ont été choisis d'après les enseignements tirés de la revue de littérature présentée en première partie d'article. Ont ainsi été d'abord évoqués le système de pilotage de l'organisation en général, les outils communément utilisés, puis le budget et la procédure budgétaire, avec une attention particulière portée aux utilisations politiques externes ainsi qu'aux utilisations instrumentales internes du budget par les associations. L'analyse qualitative des données collectées s'est inspirée des modes thématiques d'analyse du discours (Bardin, 1998). Les résultats de cette analyse ont été examinés à l'aune de la revue de littérature. 
Dans une perspective d'approfondissement des éléments recueillis, ce premier axe de collecte auprès de responsables de théâtres et d'analyse de données a été complété par un travail analogue mené auprès du président de chacun des conseils d'administration, ainsi que des mairies, après adaptation de la grille d'entretien. Ont ainsi été interviewés dans les mairies le directeur des affaires culturelles et l'élu en charge de la culture. Ces entretiens ont permis de compléter les informations recueillies lors de la première phase d'entretiens et, plus particulièrement pour les entretiens menés dans les mairies, de collecter des données sur la nature des liens entre théâtres et mairies, sur les attentes de la mairie vis-à-vis des théâtres financés par la ville, sur le rôle de la mairie dans la vie de ces théâtres et leur procédure budgétaire. Il a à chaque fois été demandé de développer plus amplement le cas du théâtre associatif concerné.

Deux cas de théâtres associatifs ont ainsi été étudiés : le théâtre Le Tibre et le théâtre Orphée ${ }^{3}$, situés dans deux villes de Province réputées pour le dynamisme de leur vie culturelle. Ces villes relèvent de deux régions différentes, elles-mêmes dynamiques sur le plan de la vie associative culturelle : elles figurent ainsi parmi les cinq régions françaises où les créations d'associations artistiques et culturelles sont les plus nombreuses ${ }^{4}$.

Ces deux organisations ont plusieurs bailleurs de fonds institutionnels, tels le ministère de la Culture et de la Communication, le Conseil Régional et le Conseil Général, ainsi que la mairie, laquelle constitue l'un de leurs principaux financeurs : elle apporte ainsi plus de $60 \%$ d'un budget global annuel de l'ordre de 5 millions au théâtre Orphée, plus de $30 \%$ d'un budget global de l'ordre de 2,5 millions au Tibre. Ces théâtres sont comparables tant par la taille de l'effectif qu'ils emploient -une quarantaine de personnes-, que par leur activité. Orphée et Le Tibre programment en effet tous deux des spectacles de théâtre, de danse et de musique, dans deux salles, à des rythmes comparables : au total, sur une année, plus d'une centaine de représentations sont ainsi proposées.

Le Tibre a été créé à la fin des années 80 . Le théâtre Orphée existe sous sa forme actuelle depuis la même époque, mais hérite d'une tradition plus ancienne puisqu'il est le théâtre historique de la ville, fondé au dix-neuvième siècle. Le Tibre et Orphée fonctionnent tous deux avec un budget voté tous les ans et présenté au conseil d'administration où siègent leurs partenaires, parmi lesquels la mairie.

La section qui suit a maintenant pour but de mettre en exergue et de discuter les enseignements de l'étude de terrain concernant la part relative des utilisations politiques du budget à destination des mairies par rapport à d'autres utilisations instrumentales internes.

\section{2- Résultats et discussion : des rôles politiques aux rôles instrumentaux du budget}

L'analyse des cas permet de faire apparaître des régularités dans les modes d'utilisation du budget dans ces associations du spectacle vivant, mais également des différences. De plus, les enseignements tirés des cas permettent d'affiner les rôles théoriques du budget tels qu'ils figurent dans la littérature: si des différences de degré sont observables entre les deux établissements, globalement les rôles politiques inscrits dans le cadre des relations avec la mairie y apparaissent bien présents, mais aux côtés de rôles instrumentaux, indépendants de ces relations et paradoxalement largement développés.

L'encadré 2 ci-après présente une synthèse des observations effectuées, d'où sont issus les éléments présentés et analysés dans cette section.

3 Pour des raisons d'anonymat, les noms ont été modifiés.

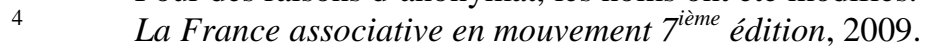


Encadré 2 : Utilisations des budgets par les théâtres

\begin{tabular}{|c|c|c|c|}
\hline \multicolumn{2}{|c|}{$\begin{array}{l}\text { Utilisations externes du budget en relation avec la } \\
\text { mairie }\end{array}$} & \multicolumn{2}{|c|}{ Utilisations instrumentales internes du budget } \\
\hline Elaboration du budget & Suivi et contrôle & Elaboration du budget & Suivi et contrôle \\
\hline \multicolumn{4}{|c|}{ Le Tibre } \\
\hline $\begin{array}{l}\text { Proposition du budget } \\
\text { global annuel au CA 4-5 } \\
\text { mois avant la fin de } \\
\text { l'année et discussion } 2 \\
\text { mois avant sa mise en } \\
\text { œuvre. } \\
\text { Généralement les actions } \\
\text { débutent avant que le } \\
\text { financement soit garanti } \\
\text { (avant les décisions des } \\
\text { collectivités). } \\
\text { Négociation des } \\
\text { subventions auprès des } \\
\text { financeurs sur la base du } \\
\text { budget. }\end{array}$ & $\begin{array}{l}\text { Re-discussion en cours } \\
\text { d'exercice du budget, qui } \\
\text { est représenté } 2 \text { ou } 3 \text { fois } \\
\text { devant le CA. } \\
\text { Explication en cas de } \\
\text { dépassement du budget. } \\
\text { Négociation éventuelle, si } \\
\text { des subventions } \\
\text { demandées pour un projet } \\
\text { validé par la collectivité } \\
\text { ont été refusées, pour } \\
\text { essayer d'obtenir leur } \\
\text { octroi. }\end{array}$ & $\begin{array}{l}\text { Préparation du budget par } \\
\text { la direction et } \\
\text { l'administration. } \\
\text { Construction du budget } \\
\text { autour des charges fixes } \\
\text { liées à l'outil et au } \\
\text { personnel ; organisation } \\
\text { par projet artistique. } \\
\text { Marge de sécurité. }\end{array}$ & $\begin{array}{l}\text { Suivi régulier du budget } \\
\text { par le service comptable } \\
\text { et l'administration } \\
\text { générale, permanent par le } \\
\text { directeur. } \\
\text { Actions correctrices par } \\
\text { des décisions qui portent } \\
\text { sur l'artistique ou sur } \\
\text { l'activité ou sur des } \\
\text { charges fixes. }\end{array}$ \\
\hline \multicolumn{4}{|c|}{ Orphée } \\
\hline $\begin{array}{l}\text { Présentation du budget au } \\
\text { CA pour approbation. } \\
\text { Explication sur la base du } \\
\text { budget au CA, à la mairie } \\
\text { et à la DRAC par le } \\
\text { directeur de ce qu'il } \\
\text { prévoit de faire. } \\
\text { Pas de négociation de la } \\
\text { subvention accordée par } \\
\text { la mairie, de montant } \\
\text { fixe. }\end{array}$ & $\begin{array}{l}\text { En cas de dépassement } \\
\text { du budget, explication et } \\
\text { argumentation par le } \\
\text { directeur et } \\
\text { l'administrateur. } \\
\text { Pas de rallonge } \\
\text { budgétaire. }\end{array}$ & $\begin{array}{l}\text { Elaboration du budget par } \\
\text { le directeur et } \\
\text { l'administrateur. } \\
\text { Construction du budget } \\
\text { autour de différents } \\
\text { pôles : une part fixe } \\
\text { (salaires...), une part } \\
\text { investissement et une part } \\
\text { artistique. } \\
\text { Marge de sécurité. }\end{array}$ & $\begin{array}{l}\text { Point sur le budget } \\
\text { mensuel entre } \\
\text { l'administrateur et le } \\
\text { directeur. } \\
\text { Suivi par le directeur et } \\
\text { l'administrateur des } \\
\text { recettes par spectacle et } \\
\text { éventuellement } \\
\text { accentuation de la } \\
\text { communication sur les } \\
\text { spectacles suivants. } \\
\text { Suivi des dépenses par les } \\
\text { responsables de service ; } \\
\text { analyse des écarts } \\
\text { éventuels et actions } \\
\text { correctrices. }\end{array}$ \\
\hline
\end{tabular}

Les cas étudiés montrent que, principalement, dans sa dimension politique, le budget est un instrument de communication de l'organisation à destination de son environnement. Au-delà, il constitue un mécanisme de rationalisation et de légitimation des actions entreprises, et éventuellement de négociation, auprès de ses partenaires institutionnels (Meyer et Rowan, 1977 ; Burchell et al., 1980), en particulier auprès de la mairie, son principal financeur.

Le budget apparaît ainsi dans ces organisations comme un instrument de communication à destination des collectivités, de la mairie en particulier, via notamment le conseil d'administration. Dans les deux associations, l'évocation du budget en conseil d'administration s'accompagne d'une communication sur la stratégie, de programmation artistique notamment : "le budget sert à expliquer ce que je veux faire » résume le directeur du Tibre. Le budget sert donc aussi de support à une communication sur la stratégie du théâtre.

Dans les deux établissements la présentation du budget constitue l'occasion de traduire les actions mises en place, de les expliquer et de leur donner du sens. Il constitue bien un outil de rationalisation des actions, qui permet de justifier et légitimer les actions entreprises comme le soulignent Meyer et Rowan (1977) ou encore Burchell et al. (1980). Ce mécanisme de 
légitimation joue selon différentes modalités. Au théâtre Orphée, s'il y a bien présentation, explication, il n'y a pas de vrai dialogue. L'un des répondants d'ailleurs perçoit en partie le conseil d'administration comme « une simple chambre d'audition »; toutefois, la volonté de vraiment expliquer, jointe à la volonté manifestée par l'auditoire de vraiment comprendre («ce sont des gens qui s'intéressent à la marche du théâtre donc il faut les mettre dans le coup »), ne permettent pas de conclure à une utilisation strictement rituelle du budget au sens de la théorie néo-institutionnelle. Au Tibre, un véritable dialogue s'engage: «le budget global annuel prévisionnel est proposé au CA 4-5 mois avant la fin de l'année et il est discuté 2 mois avant sa mise en euvre. ».

Le processus budgétaire, les présentations et explications qui l'accompagnent, constituent un mécanisme de légitimation auprès des apporteurs de capitaux, dont la présence et le soutien sont essentiels au fonctionnement des organisations culturelles (Chatelain-Ponroy, 2008). Ils permettent en particulier de justifier en phase de prévision les subventions à verser ainsi que leur utilisation et éventuellement, comme au Tibre, de négocier avec les financeurs publics ces subventions : "Le budget élaboré deux mois avant l'exercice va servir de demande de financement ». L'information budgétaire sert ainsi de "plaidoyer politique externe », comme le suggèrent Covaleski et Dirsmith (1983) à propos de l'utilisation du budget dans un cadre institutionnel, qui est celui des organisations étudiées. Au théâtre Orphée en revanche, point de négociation en phase de constitution du budget auprès de la mairie : "la subvention est stable depuis plusieurs années : elle est tellement importante qu'elle ne peut pas augmenter».

La dimension politique est également présente en phase de suivi, lorsque se produisent d'éventuels dépassements budgétaires. Il s'agit à nouveau de fournir des comptes en vue notamment de légitimer le besoin de ressources, lequel est essentiel à la survie de l'organisation (Meyer et Rowan, 1977). Ainsi, les éventuels écarts sont expliqués aux partenaires des deux théâtres. Au théâtre Orphée, l'explication et l'argumentation ne s'accompagnent pas de négociation : il n'est pas envisageable de demander lors de cette phase d'éventuelles rallonges budgétaires, la subvention préalablement accordée par la mairie étant déjà de montant conséquent: "il n'y a pas de rallonge sur les subventions en cours d'année ». Au Tibre, l'explication peut s'accompagner d'une négociation dans la mesure où les écarts éventuellement constatés s'expliquent non par des erreurs dans la prévision des charges ou de la billetterie, mais par le fait que des subventions demandées à une collectivité partenaire n'ont finalement pas été accordées. Le théâtre peut alors souligner que ces subventions étaient destinées à financer un projet justement validé par la collectivité, sur lequel des charges ont déjà été engagées, afin d'essayer d'obtenir l'octroi de la subvention. Toutefois, il n'y a pas de demande de subvention supplémentaire par rapport à celles effectuées au préalable.

L'ensemble de ces observations a permis de souligner une utilisation politique du budget, laquelle s'exprime différemment suivant les établissements. L'absence au théâtre Orphée de l'utilisation du budget pour la négociation auprès du principal financeur conduit à conclure à des utilisations politiques du budget moins développées dans ce théâtre qu'au Tibre. Qu'en est-il alors de l'utilisation instrumentale, et dans une perspective interne, du budget?

Alors que l'on pouvait, suite aux limites théoriques évoquées en première partie d'article, s'attendre à une fonction instrumentale interne du budget relativement restreinte, nous avons pu observer paradoxalement une utilisation du budget marquée par un besoin de suivi de l'activité et des résultats en interne, le tout dans une perspective de contrôle et de surveillance de l'activité.

Tout d'abord, la prévision apparaît comme instrumentale parce qu'elle pose les bases du système de suivi et de contrôle des résultats. En phase de prévision, la primauté de la 
dimension artistique sur la gestion semble claire dans les deux cas : "au départ il y a une couleur artistique, à partir de là un budget artistique » déclare ainsi l'administrateur d'Orphée. L'on retrouve bien le principe classique d'élaboration des budgets suivant lequel l'outil traduit les plans d'action, ainsi que la ou les missions de l'organisation (Bouquin, 1998).

Toutefois, la problématique de la prévision artistique (Chiapello, 1997, 1999) déjà évoquée dans la partie théorique de cet article revêt des formes différentes dans les organisations étudiées. Au Tibre en effet, même si l'on déclare essayer en phase de prévision "de prévoir le mieux possible les risques », cette prévision paraît difficile, non pas tant du fait de la nature des produits en tant que telle mais du fait des financements publics paradoxalement à l'origine d'une incertitude fragilisatrice : "Quand on élabore le budget, quand on étale les charges on n'a pas de problème [...]! Pour les charges artistiques on a des outils qui nous permettent d'évaluer, des habitudes. [...] Idem pour les charges de structure. [...] La difficulté, ce sont les financements publics : on fait des demandes et on n'a pas toujours la réponse » explique l'administrateur du Tibre. Pour faire face à cette incertitude, "les charges sont prévues un peu en dessus, les produits un peu en dessous » et les prévisions ensuite ajustées en fonction des nouvelles informations: le budget n'est pas figé, jusqu'au dernier trimestre. Le théâtre a également tendance à "freiner [a priori] son activité » pour anticiper le fait que certaines demandes de subventions recevront in fine des réponses négatives. Au théâtre Orphée en revanche, la question de la prévision des coûts et des financements ne semble pas être vécue comme problématique. L'administrateur d'Orphée souligne d'ailleurs que "le social est difficile mais pas le niveau comptable et budgétaire »; selon lui, le fait que l'essentiel du financement soit constitué par une subvention de montant fixe systématiquement reconduite réduit considérablement l'incertitude, de même que l'existence d'une convention triennale entre le théâtre et la ville. Les recettes des spectacles ont donc un rôle marginal dans le financement du théâtre ; l'expérience permet par ailleurs à l'administrateur de les évaluer avec une relative précision, de même que la fréquentation des spectacles et les coûts associés, mais aussi d'anticiper le montant des autres charges telles celles liées aux fournisseurs ou au personnel. In fine, il semble possible de parvenir à «d'assez bonnes prévisions». Pour faire face à l'incertitude qui subsiste toutefois, une petite marge de manœuvre est prévue : par exemple, "les recettes sont plutôt prévues un peu en deçà ».

Finalement, sur les deux cas étudiés, il apparaît, de façon paradoxale et surprenante, que le budget joue un rôle en matière d'allocation des ressources et de prévision. La question qui se pose dès lors concerne l'utilisation qui est faite en interne de ces prévisions. Paradoxalement, malgré les difficultés éventuellement associées à ces prévisions, les interviewés s'appuient sur un suivi précis des réalisations comparativement au budget. Cela correspond à un besoin de suivi interne des budgets, lequel semble lié à un souci de suivi et de pilotage stratégique de l'activité, qui permet aux organisations de savoir " où elles en sont » et si elles vont pouvoir mener à bien leurs objectifs de programmation, mais aussi à une volonté de "rester dans les clous » du budget. Ainsi, des vérifications et contrôles sont mis en œuvre sur la base d'éléments qui composent le budget. Par exemple, au Tibre sont évoqués un suivi "régulier» par l'administrateur et un suivi «permanent» par le directeur. De même au théâtre Orphée, un «point budget mensuel » est effectué par l'administrateur et le directeur. Les réalisations sont comparées aux prévisions et, des écarts éventuellement constatés, découlent des actions correctrices. En ce sens, les budgets sont bien, en phase de pilotage, à l'origine d'une tension incitatrice. Ainsi, le directeur du théâtre Orphée explique que, "si sur un spectacle donné on n'a pas les recettes escomptées alors ça peut déclencher des actions de communication, de relations publiques sur les spectacles suivants ». Quant aux dérives sur les coûts, elles sont rarissimes, les responsables de service s'organisant en interne, effectuant des arbitrages, pour respecter leur budget. Si une dérive se profile toutefois, par exemple si la mise en scène d'un 
spectacle est conditionnée par l'engagement de frais supplémentaires, cette dérive est signalée par le responsable de service ; une réunion entre ce dernier, l'administrateur et le directeur permet alors d'analyser la cause de l'écart et éventuellement de mettre en place des actions correctrices. Au Tibre, la constatation d'écarts entraîne également des actions correctrices : "on réajuste par des décisions qui portent sur l'artistique ou sur l'activité ou sur des charges fixes ». Par exemple, le programme n'est pas définitivement arrêté : certaines choses seront faites ou non suivant les écarts éventuellement constatés.

Finalement, on observe dans ces organisations la mise en place d'un suivi des résultats par rapport au budget fondé sur un calcul d'écarts, ainsi que l'utilisation des résultats diagnostiqués pour prendre des décisions en matière d'ajustement et pour mener à bien des actions correctrices. Dans les deux établissements étudiés, si à la base le budget mis en œuvre répond à la nécessité de communiquer avec le financeur public, il joue aussi un rôle interne dans le sens où il permet le calcul et le suivi d'écarts nécessaires à la mise en œuvre de révisions budgétaires et d'actions correctrices en cours de période.

\section{Conclusion}

Cet article visait à répondre à la question de recherche suivante : parmi les utilisations du budget par les associations du spectacle vivant, quelles sont celles qui relèvent d'un usage politique externe dans le cadre des relations avec les collectivités, quelles sont celles qui correspondent à un usage instrumental interne et quelle est la part relative de ces différents types d'utilisations?

La revue de littérature a permis de poser le cadre théorique de l'étude de terrain et donc de faire émerger une conception d'un budget théorique avant tout politique à visée externe et dont la mise en œuvre en interne des fonctions instrumentales serait théoriquement problématique au sein des associations du spectacle vivant, du fait des spécificités de ces dernières.

Le protocole de recherche utilisé pour l'étude de terrain complétant la revue de littérature s'est appuyé sur des entretiens semi-directifs menés auprès des responsables de deux théâtres associatifs à financement principalement municipal, des présidents des conseils d'administration de ces théâtres ainsi qu'auprès de responsables culturels dans les mairies concernées (directeurs des affaires culturelles, élus en charge de la culture).

L'étude de terrain a permis de compléter et d'affiner la conception théorique des rôles du budget au sein des associations du spectacle vivant qui avait émergé de la revue de littérature. A l'issue de la phase empirique se distinguent des utilisations politiques externes du budget, inscrites dans le cadre des relations avec la mairie, ainsi que des utilisations instrumentales internes, indépendantes de ces relations. L'importance relative de la dimension politique externe du budget par rapport à sa dimension instrumentale interne varie suivant les établissements : ainsi, le poids relatif de la dimension politique est plus faible au théâtre Orphée qu'au Tibre. Dans les deux théâtres toutefois, si la dimension politique externe apparaît bien présente conformément à ce que l'on pouvait attendre au vu de la littérature, la dimension instrumentale interne apparaît quant à elle plus prégnante que dans la littérature. Tout se passe comme si ces associations du spectacle vivant se réappropriaient l'outil initialement politique qu'est le budget, né de la relation avec la mairie et plus généralement avec les collectivités, pour véritablement s'en servir en interne à des fins instrumentales. De ces résultats découlent les prolongements de recherche possibles, parmi lesquels la réplication de cette étude dans d'autres théâtres associatifs à des fins de validation, mais aussi dans le but de faire émerger des facteurs explicatifs des différences constatées ; une extension de l'étude à d'autres types d'associations du spectacle vivant, impliquant éventuellement d'autres 
collectivités, pourrait également être pertinente. Enfin, des mécanismes de couplage (Covaleski et Dirsmith, 1983, 1988a, 1988b) entre les utilisations politiques externes et les utilisations instrumentales internes du budget apparaissant en filigrane dans les résultats présentés ici, l'étude de ces derniers constituerait une autre piste de recherche intéressante.

\section{Bibliographie}

Abdallah C. (2007), "Production et appropriation du discours stratégique dans une organisation artistique », Revue Française de Gestion, Vol. 33, N174, p. 61-76, mai.

Abrahamson E. (1996), « Management Fashion », Academy of Management Review, Vol. 16, p. 586-612.

Agid P., Tarondeau J.C. (2003), «Manager les activités culturelles », Revue Française de Gestion, Vol. 29, №142, p. 103-112, janvier.

Amans P., Rascol-Boutard S. (2008), «La performance entre construit social et indicateur simplifié », Finance-Contrôle-Stratégie, Vol. 11, №3, p. 45-63.

Amans P., Villesèque-Dubus F. (2008), «Le budget : fonctions théoriques et application au cas d'une organisation du spectacle vivant », Revue Sciences de Gestion, N67, p. 61-78.

Anthony R.N. (1965), Planning and Control Systems: a Framework for Analysis, Harvard University Press.

Aristote (1995), La Politique, trad. J. Tricot, Vrin, Bibliothèque des textes philosophiques, Paris.

Assassi I. (2003), «Spécificités du produit culturel : L'exemple du spectacle vivant », Revue Française de Gestion, Vol. 29, N¹42, p. 129-146, janvier.

Assassi I. (2008), «Régulation du canal dans un environnement incertain: Le cas des arts du spectacle », Revue Française de Gestion, Vol. 34, N¹82, p. 135-153, mars.

Bardin L. (1998), L'analyse de contenu, PUF, Le psychologue, $9^{\mathrm{e}}$ édition.

Bathurst R., Williams L., Rodda A. (2007), «Letting Go of the Reins: Paradoxes and Puzzles in Leading an Artistic Enterprise », International Journal of Arts Management, Vol. 9, $\mathrm{N}^{\circ} 2$, p. 29-38, Winter.

Benghozi P.J. (1990), «Les feux de la rampe, gestion et régulation des activités culturelles », Cahiers de recherche du CRG, École Polytechnique, Paris, p. 7-20.

Berland N. (1999), «L'histoire du contrôle budgétaire en France. Les fonctions du contrôle budgétaire, influences de l'idéologie, de l'environnement et du management stratégique », Thèse en sciences de gestion, Université Paris-Dauphine.

Bonnafous-Boucher M., Chatelain-Ponroy S., Evrard Y., Mazallon F. (2003), «Quel avenir pour les théâtres lyriques ? »Revue Française de gestion, Vol. 29, N 142, p. 169-188.

Bouquin H. (1998), Le contrôle de gestion, PUF, $5^{\mathrm{e}}$ édition.

Bruns W.J., Waterhouse J.H. (1975), «Budgetary Control and Organization Structure», Journal of Accounting Research, p. 177-203, Autumn.

Burchell S., Clubb C., Hopwood A., Hugues J., Nahapiet J. (1980), "The Roles of Accounting in Organizations and Society », Accounting Organizations and Society, Vol. 5, $\mathrm{N}^{\circ} 1$, p. 5-27.

Burlaud A., Simon C. (2000), Le contrôle de gestion, Repères, La Découverte.

Chatelain S. (1998), « Du budget administratif au budget outil de gestion : Le cas des musées français », Finance-Contrôle-Stratégie, Vol. 1, №3, p. 5-33, septembre.

Chatelain-Ponroy S. (2001), « Difficultés d'analyse des coûts des organisations culturelles », $X X I I^{e}$ Congrès de l'AFC, Metz, 17-19 mai.

Chatelain-Ponroy S. (2003), «Prolégomènes à l'analyse des coûts dans des organisations culturelles municipales », Comptabilité-Contrôle-Audit, Tome 9, Vol. 1, p. 79-93, mai.

Chatelain-Ponroy S. (2008), «Le contrôle de gestion dans des bureaucraties professionnelles non lucratives : Une proposition de modélisation », Mémoire d'HDR, CNAM. 
Chekkar R., Zoukoua E.A. (2009), « Comportement des audités dans le processus d'audit : Le cas du contrôle des associations par les financeurs publics », $X X X^{e}$ Congrès de l'AFC, Strasbourg, mai.

Chiapello E. (1994), «Les modes de contrôle des organisations artistiques », Thèse en sciences de gestion, Université Paris IX-Dauphine.

Chiapello E. (1997), «Les organisations et le travail artistiques sont-ils contrôlables ? 》 Réseaux, №86, p. 77-113, novembre-décembre.

Chiapello E. (1998), Artistes versus Managers : Le management culturel face à la critique artistique, Editions Métailié.

Chiapello E. (1999),. « Art, innovation et management : Quand le travail artistique interroge le contrôle », In Questions de contrôle (Eds, Collins, L.), PUF, Gestion, p. 194-218.

Covaleski M.A., Dirsmith M.W. (1983), «Budgeting as a Means for Control and Loose Coupling », Accounting Organizations and Society, Vol. 8, 4, p. 323-340.

Covaleski M. A., Dirsmith M. W. (1986), «The Budgetary Process of Power and Politics », Accounting, Organizations and Society, Vol. 11, №3, p. 193-214.

Covaleski M. A., Dirsmith M. W. (1988a), «The Use of Budgetary Symbols in the Political Arena: A Historically Informed Field Study », Accounting, Organizations and Society, Vol. $13, \mathrm{~N}^{\circ} 1$, p. 1-24.

Covaleski M.A., Dirsmith M.W. (1988b), «An Institutional Perspective on the Rise, Social Transformation, and Fall of a University Budget Category », Administrative Science Quarterly, 33, p. 562-587.

DiMaggio P.J., Powell W.W. (1983), «The Iron Cage Revisited: Institutional Isomorphism and Collective Rationality in Organizational Fields », American Sociological Review, Vol. 48, p. 147-160.

Evrard Y., Chiapello E. (2004). «Introduction». In Le management des entreprises artistiques et culturelles (Eds, Evrard Y.), Economica Gestion, $2^{\mathrm{e}}$ édition, p. 5-12.

Fabre P. (2004), «Le contrôle des associations par les mairies françaises: Un défi à la gouvernance des villes», In Management local, de la gestion à la gouvernance : VI rencontres Ville-Management, Dalloz, p. 233-258.

Fabre P. (2005a), «L'évaluation de la performance des associations dans les villes françaises, entre proximité et contingence », Comptabilité-Contrôle-Audit, Vol. 11, No 1, p. 55-77, mai.

Fabre P. (2005b), «Le contrôle des associations par les mairies françaises: Etude comparative portant sur les secteurs sports, culture et prévention de la délinquance », Thèse en sciences de gestion, Université d'Orléans.

Fabre P. (2006), «Les associations face aux communes: quels leviers d'actions pour l'appropriation des ressources?», Journée de Recherche "Quel management pour les associations? », Tours, 12 janvier.

Gehrke I., Zarlowski P. (2003), «La diffusion de la valeur actionnariale en France: Une lecture néo-institutionnelle », Comptabilité-Contrôle-Audit, numéro spécial, p. 189-205, mai.

Gervais M. (1997), «Système budgétaire et animation des hommes », In Encyclopédie de gestion (Eds, Joffre P., Simon Y.), Economica, Paris, p. 3174-3199.

Gignon-Marconnet I. (2003), «Les rôles actuels de la gestion budgétaire en France : une confrontation des perceptions de professionnels avec la littérature », Comptabilité-ContrôleAudit, Tome 9, $\mathrm{N}^{\circ}$ 1, p. 53-78, mai.

Glaser B.G., Strauss A. (1967), The Discovery of Grounded Theory: Strategies for Qualitative Research, Aldine de Gruyter, New York.

Lande E. (2001), «Le contrôle externe des associations par les organismes de financement », $X X I I^{e}$ Congrès de l'AFC, Metz, 17-19 mai.

Le Theule M.A. (2008), "Comptabilité et contrôle de gestion dans les organisations créatives : Une gestion des possibles ? », Thèse en sciences de gestion, CNAM. 
Meyer J.W., Rowan B. (1977), «Institutional Organizations: Formal Structure as Myth and Ceremony », American Journal of Sociology, 83, p. 340-363.

Meyer J.W., Rowan B. (1978). The Structure of Educational Organizations, In Environments and Organizations (Eds, Meyer M.W. et. al.), Jossey-Bass.

Ragi T. (2000), «Les relations entre associations et pouvoirs publics », Revue Projet, $\mathrm{N}^{\circ} 264$, décembre.

Rojot J. (2005), Théorie des organisations, Paris, ESKA, 2 édition.

Simons R. (1990), «The Role of Management Control Systems in Creating Competitive Advantage: New Perspectives ». Accounting Organizations and Society, Vol. 15, N¹-2, p. 127-143.

Simons R. (1995), Levers of Control. Harvard Business School Press.

Sponem S. (2004), «Diversité des pratiques budgétaires des entreprises françaises: Proposition d'une typologie et analyse des déterminants », Thèse en sciences de gestion, Université Paris-Dauphine.

Yin R.K (1990), Case study research, Sage publications.

Zoukoua E.A. (2006), «Associations : Un défi pour les théories de la gouvernance?», Journée de Recherche "Quel management pour les associations? », Tours, 12 janvier.

Zoukoua E.A. (2008), "Quelle gouvernance pour les organisations du tiers secteur? Le cas des associations loi 1901 », Thèse en sciences de gestion, Université d'Orléans. 